\title{
Real-Time Trajectory Optimization for Noise Abatement of Helicopter Landings*
}

\author{
Takeshi TSUCHIYA**, Hiroshi IKAIDA**, Hirokazu ISHII***, \\ Hiromi GOMI*** and Yoshinori OKUNO*** \\ **University of Tokyo, \\ 7-3-1 Hongo, Bunkyo-ku, Tokyo, Japan \\ E-mail: tsuchiya@mail.ecc.u-tokyo.ac.jp \\ ***Japan Aerospace Exploration Agency, \\ 6-13-1 Osawa, Mitaka, Tokyo, Japan
}

\begin{abstract}
In this study, an optimization method is developed to reduce the ground noise impact of helicopter approach flights, and flight experiments are conducted to confirm the effectiveness of this optimization. First, we build a noise model of the JAXA (Japan Aerospace Exploration Agency) experimental helicopter based on measurement results. Then, we define optimal control problems to minimize the noise levels measured at points on the ground surface. The main objective of this paper is to propose a real-time flight trajectory optimization method for solving the optimization problems using actual flight conditions with an on-board computer. To validate the effect of the proposed method, some numerical simulations are conducted under real flight experiment scenarios. The obtained optimal solutions are characterized by steep flight path angles so as to avoid the flight conditions that generate large noise, avoidance of the measurement points, and short flight times; these optimal flights are different from conventional flight trajectories. Finally, the validity of the proposed optimization method is examined by means of flight experiments with the experimental JAXA helicopter. The actual flight experiments result in noise reduction, and prove the effectiveness of the optimization.
\end{abstract}

Key words: Aerospace Engineering, Optimal Control, Acoustic Techniques

\section{Introduction}

Helicopters have specific maneuvering capabilities, such as hover and vertical take-off and landing, which enable them to conduct various operations that are not feasible with general fixed-wing aircraft, and make them an effective means of transport in suburban and mountainous areas. Although helicopters are used for live broadcasts, life-saving services, rescue missions, and various other operations, their use is still limited at present. One of the main obstacles to the widespread use of helicopters is the loud noise they generate. While there are many noise sources in a helicopter, the blade-vortex interaction (BVI) noise is known as the most annoying sound during landing approaches. BVI noise is caused by impulsive pressure fluctuations on the main rotor blades, induced by tip vortices shed by the preceding blades. During a conventional approach, the tip vortices pass close to the following blades and consequently a strong BVI noise is generated. Meanwhile, in actual flights, selection of a landing approach path is based on the visual flight rule (VFR) and is basically entrusted to the pilot's manual control. When flying over densely populated areas, pilots have to consider both ride quality and ground noise impact. However, since the noise level and propagation characteristics easily change due to factors such as flight and 
atmospheric conditions, the actual noise level at the ground is not necessarily consistent with the sound anticipated by the pilot. Additionally, the more unfamiliar the pilots are with the landscape, the higher the possibility of excess noise pollution. It has been suggested the noise impact be minimized by its dispersion over areas that are tolerant of noise such as industrial zones, rivers, forests and highways using a precision approach system ${ }^{(1)}$.

In this context, NASA established a Rotorcraft Noise Model (RNM) that estimates noise levels three-dimensionally ${ }^{(2)}$. Since this RNM was built from actual flight experiment data, the noise levels around a helicopter could be predicted in real-time with computers. However for simplification, this modeling excludes various dynamic parameters (e.g., the tip-pass-plane angle of the main rotor and acceleration) that affect the magnitude of the BVI noise level. As a result, the predictions are not very consistent with the observed ground noise level. Instead, the more practical Quasi-static Acoustic Sound Mapping (Q-SAM) is preferable for helicopter noise estimation ${ }^{(3)}$. Q-SAM includes variable parameters that are critical for more accurate prediction. Its application to the noise abatement optimal control problem in numerical simulations has shown its effectiveness and feasibility ${ }^{(4)}$. In Japan, the Japan Aerospace Exploration Agency (JAXA) has performed advanced studies on methods for measuring noise using actual aircraft, and has measured the noise at ground level and on board an experimental helicopter, the MuPAL- $\varepsilon$ (Fig. 1) $)^{(5)(6)}$.MuPAL" is an abbreviation for Multi-Purpose Aviation Laboratory. MuPAL- $\varepsilon$, which is a helicopter based on the Mitsubishi MH2000A, is equipped with a precision data acquisition system, programmable cockpit displays, and other experimental support systems ${ }^{(7)}$.

The University of Tokyo is currently pursuing collaborative research with JAXA regarding landing approach trajectories that minimize helicopter noise damage. In previous studies ${ }^{(8)(9)}$, JAXA built a noise model of MuPAL- $\varepsilon$ which depends on the flight path angle and bank angle, and University of Tokyo defined optimal control problems that minimized the noise levels measured at points on the ground surface, and obtained optimal controls for some flight constraints and wind conditions. The validity of the optimal flight patterns was

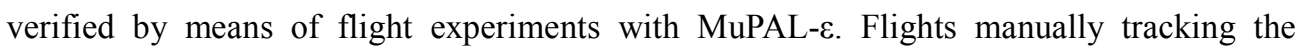
pre-calculated optimal trajectories resulted in lower noise impact on the ground than conventional flights. Detailed examinations of these actual flight experiments, however, revealed that there were some results that did not show the predicted optimization effects because of unexpected external disturbances (i.e., variation of wind and pilot's tracking error) during flight experiments. To resolve these problems and improve the practicality of our system, we have developed an optimization algorithm that does not simply optimize the trajectory before the flight, but rather optimizes it during the flight, based on actual conditions. In other words, this study focuses on a real-time flight trajectory optimization using an on-board PC.

The next section defines the three-dimensional optimal control problem in which the helicopter engages in three-dimensional flight. The problem definition accords with a flight test environment. This paper improves the noise model from the results of Ref. 0 , and attempts to verify the validity of this improved model. An algorithm for real-time flight trajectory optimization is proposed, and the real-time optimization and offline optimization are compared in numerical simulations. Finally, actual flight experiments prove the effectiveness of the real-time optimization and the noise abatement of flight paths based on the optimal solutions.

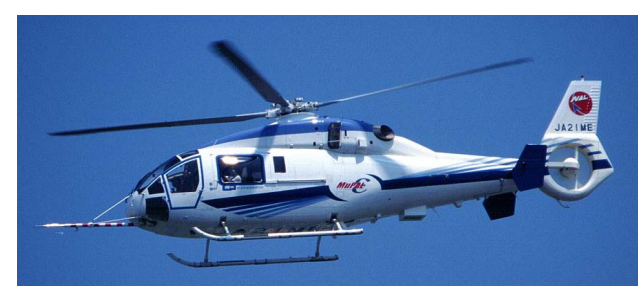

Fig. 1 The JAXA experimental helicopter, MuPAL- $\varepsilon$. 


\section{Nomenclature}

att : total noise attenuation

$a t t_{\text {air }}, a t t_{\text {dist }}$ : noise attenuation by atmospheric absorption and distance

$E_{A}, E_{A i}$ : energy-based noise estimation (at measurement point $i$ )

$g:$ acceleration due to gravity

$h, h_{\mathrm{f}}$ : altitude, final altitude

$J$ : performance index

$L, \quad L_{i}$ : noise level (at measurement point $i$ )

$L_{\text {allow } i}$ : noise allowance at measurement point $i$

$L_{A}$ : temporal-mean noise level

$L_{m}:$ measurement-point mean noise level

$L_{\text {ref }}$ : reference noise level (noise level measured at reference distance $r_{\text {ref }}$ )

$m:$ mass

$N_{1}, N_{2}:$ the numbers of nodal points

$r, r_{i}$ : distance from helicopter (to measurement point $i$ )

$r_{\text {ref }}$ : reference distance at which the noise level $L_{\text {ref }}$ is measured

$S_{u}$ : square integration of variation of control values

$t, t_{\mathrm{f}}$ : time, final time

$T:$ thrust

$T_{\text {final }}$ : final search time range

$T_{\text {overlap }}$ : overlapping time between adjacent stages

$T_{\text {stage }}:$ duration of each stage

$T_{\text {update }}$ : time interval to update the optimal result

$u_{g}, \psi_{g}$ : wind speed, wind direction

$U, W$ : horizontal velocity, climb rate

$w$ : weight factor for $S_{u}$ in the performance index

$x, y$ : position of helicopter ( $x$ and $y$ coordinates)

$\gamma:$ flight path angle

$\gamma_{2}$ : flight path angle modified by horizontal acceleration

$\Phi, \psi, \Theta$ : roll angle, heading angle, pitch angle

\section{Problem Definition}

\subsection{Helicopter Dynamics Model}

The motion of the helicopter represented by a point mass was described by the following differential equations:

$$
\begin{aligned}
& \dot{x}=U \cos \psi-u_{g} \cos \psi_{g} \\
& \dot{y}=U \sin \psi-u_{g} \sin \psi_{g} \\
& \dot{h}=W \\
& \dot{U}=-\frac{T}{m} \cos \Phi \sin \Theta \\
& \dot{W}=-g+\frac{T}{m} \cos \Phi \cos \Theta \\
& \dot{\psi}=\frac{T}{m U} \sin \Phi
\end{aligned}
$$

The NED (North East Down) coordinate system was defined to express the position of the helicopter, but the vertical position was expressed in terms of the altitude $h$ instead of the z-axis. The six state variables were controlled by the thrust $T$, pitch angle $\Theta$, and roll angle $\Phi$. It was assumed that the mass $m$ did not change while flying. In these equations 
of motion, only two external forces act on the helicopter: the thrust of the main rotor and gravity. The equations included a horizontal wind with the wind velocity $u_{g}$ and wind direction $\psi_{g}$.

In addition, constraint conditions during the flight were defined. The helicopter motion was calculated using the above-mentioned simple model, but the actual helicopter was controlled by the pilot manually during the flight experiments. It was therefore necessary for the pilot to follow the optimal solutions without being burdened by an excessive workload. An experiment using a flight simulator owned by JAXA was performed to determine the constraint conditions, and then settled upon the four types of constraint conditions listed below.

- Horizontal-velocity limit

$$
50 \leq U \leq 100 \mathrm{kt}
$$

- Climb rate limit

Considering the psychological impact on a pilot of taking a steep dive at low altitudes, a relatively strict lower limit is established at low altitudes. For the upper limit on the climb rate, the helicopter will descend consistently in the landing approach. When $h \geq 480 \mathrm{ft}$,

$$
-800 \leq W \leq 0 \mathrm{ft} / \mathrm{min} .
$$

When $h \leq 480 \mathrm{ft}$,

$$
-\left(\frac{h}{480}\right) 800 \leq W \leq 0 \mathrm{ft} / \mathrm{min} .
$$

- Roll angle limit

The roll angle is limited depending on the altitude as in the case of the climb rate. When $h \geq 1000 \mathrm{ft}$,

$$
-10 \leq \Phi \leq 10 \mathrm{deg} .
$$

When $h \leq 1000 \mathrm{ft}$,

$$
-\left(\frac{h}{1000}\right) 10 \leq \Phi \leq\left(\frac{h}{1000}\right) 10 \mathrm{deg} .
$$

- Acceleration limit

$$
\begin{aligned}
& -1.5 \leq \dot{U} \leq 0 \mathrm{kt} / \mathrm{s} \\
& -100 \leq \dot{W} \leq 100 \mathrm{ft} / \mathrm{min} / \mathrm{s}
\end{aligned}
$$

\subsection{Noise source model}

JAXA has measured the noise level of MuPAL- $\varepsilon^{(5)(6)}$. The measurement shows that the noise, especially BVI noise, depends on the flight path angle and airspeed and has directivity of radiation. However, a characteristic of the noise of MuPAL- $\varepsilon$ is that the variations caused by the airspeed are smaller than those by the flight path angle. Moreover, though the helicopter noise has the directivity, it was ignored for fast computation in this study. This study utilized the noise source model of MuPAL- $\varepsilon$, which depends on the flight path angle $\gamma\left(=\tan ^{-1} W / U\right)$, the roll angle $\Phi$, and the acceleration $\dot{U}$. The noise is largest around a flight path angle of -5 deg because of the BVI noise. An ascent flight that requires large engine torque also generates a large noise. The noise level also depends on the roll angle and acceleration because a roll flight and an acceleration flight require large engine torque and change the distance between the main rotor and the tip vortices. Figure 2 shows a noise source model with a reference noise level, $L_{\text {ref }}$, which was measured at a distance of $100 \mathrm{~m}$ from the helicopter (reference distance $r_{\text {ref }}$ ). The noise level 


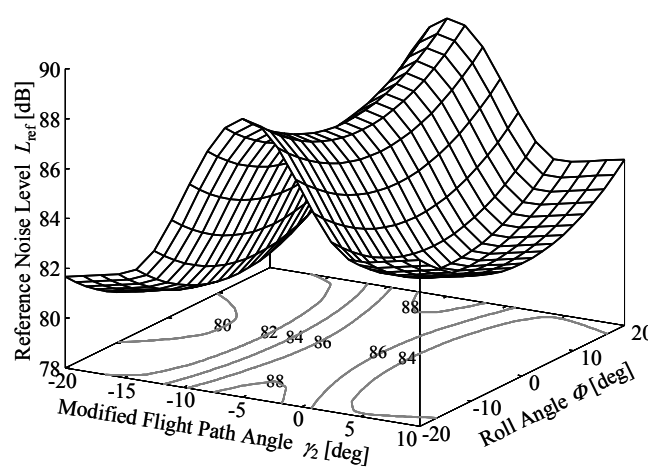

Fig. 2 Noise source model.

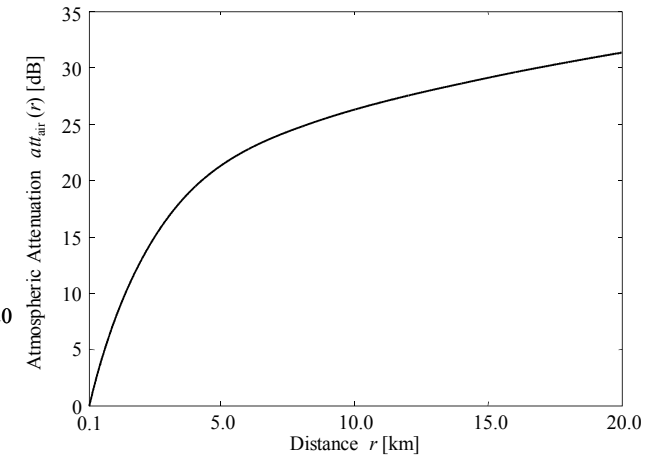

Fig. 3 Atmospheric absorption model.

compensates for human hearing using A-weighting. The noise level $L_{\text {ref }}$ is expressed in terms of the following equations. When $\gamma_{2}<-15 \mathrm{deg}$ or $5 \mathrm{deg}<\gamma_{2}$,

$$
L_{\text {ref }}\left(\gamma_{2}, \Phi\right)=81.5+0.1 \gamma_{2}-80 \log (\cos \Phi) \quad[\mathrm{dB}] .
$$

When $-15 \leq \gamma_{2} \leq 5 \mathrm{deg}$,

$$
L_{\mathrm{ref}}\left(\gamma_{2}, \Phi\right)=84.5+0.1 \gamma_{2}+3 \cos \left(\frac{\gamma_{2}+5}{10} \pi\right)-80 \log (\cos \Phi) \quad[\mathrm{dB}] .
$$

Here, $\gamma_{2}$ is a modified flight path angle expressed as

$$
\gamma_{2}=\gamma+\frac{180}{\pi} \cdot \frac{\dot{U}}{g} \quad[\mathrm{deg}] .
$$

\subsection{Attenuation model}

The noise emitted from the helicopter is subject to the influence of various factors during its propagation to ground level. This paper considers two types of attenuation: the inverse square law of distance and atmospheric absorption. For the inverse square law of distance, the attenuation $a t t_{\text {dist }}(r)$ at a point distance $r$ from the noise source is

$$
\operatorname{att}_{\mathrm{dist}}(r)=20 \log \frac{r}{r_{\text {ref }}} \quad[\mathrm{dB}] .
$$

For the atmospheric absorption, the relation between the distance and overall noise level depends on the frequency characteristics of the noise source, because the atmospheric absorption depends on frequency. The attenuation coefficient is given as a function of the frequency and the temperature, humidity and pressure of the air in Ref. 10. The attenuation coefficient and A-weighting were calculated for the one-third octave band noise level of MuPAL- $\varepsilon$ and added to the spectrum to obtain one-third octave spectra at various distances. Then, the overall noise levels were obtained by energetic summation of all the bands. Figure 3 shows the relation between the atmospheric absorption $a t t_{\text {air }}(r)$ and the distance $r$ from MuPAL- $\varepsilon$. The two attenuations are combined to compute att $(r)$.

$$
\operatorname{att}(r)=a t t_{\text {dist }}(r)+a t_{\text {air }}(r) \quad[\mathrm{dB}]
$$

According to Eqs. (14) - (18) at time $t$, the noise level $L(t)$ measured at a distance $r(t)$ from the helicopter is calculated as

$$
L(t)=L_{\mathrm{ref}}\left(\gamma_{2}(t), \Phi(t)\right)-\operatorname{att}(r(t)) \quad[\mathrm{dB}] .
$$

This study ignored the time needed for the noise to propagate from the noise source to the measurement point. The noise source model and attenuation model indicated herein are simple. However, the excessive complication of these models increases the computation 


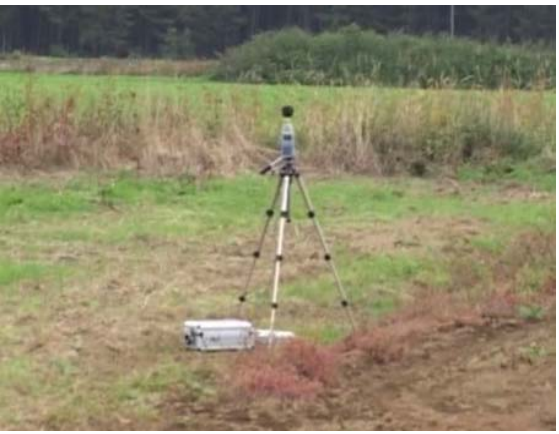

Fig. 4 A noise measurement device.

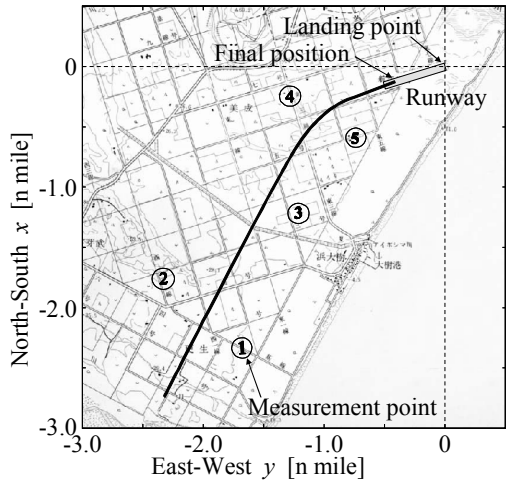

Fig. 5 A reference path and five measurement points.

Table 1 The coordinates of measurement points

\begin{tabular}{|c|c|c|c||c|c|}
\hline $\begin{array}{c}\text { Number of } \\
\text { measurement points }\end{array}$ & $x, \mathrm{n}$ mile & $y, \mathrm{n}$ mile & $h, \mathrm{ft}$ & Variable & Final condition \\
\hline 1 & -2.37 & -1.66 & -11.8 & $x, \mathrm{n}$ mile & -0.14 \\
\hline 2 & -1.79 & -2.40 & 18.4 & $y, \mathrm{n}$ mile & -0.45 \\
\hline 3 & -1.26 & -1.30 & -11.4 & $h$ & $h_{\mathrm{f}}$ \\
\hline 4 & -0.36 & -1.36 & 2.1 & $W, \mathrm{kt}$ & 50 \\
\hline 5 & -0.65 & -0.90 & -9.7 & $\Psi, \mathrm{ft} / \mathrm{min}$ & free \\
\hline
\end{tabular}

cost and takes a lot of time for convergence in the real-time optimization.

\subsection{Flight test conditions}

Initial conditions, terminal conditions, and a performance index of the optimal control problems were defined according to the flight test environment. This subsection summarizes the flight test conditions. The flight tests were carried out in the vicinity of Taiki Multi-Purpose Aeronautical Park in Hokkaido at latitude 42.50 degrees north and longitude 143.44 degrees east in Japan. The reference flight path for approaching the runway was determined with consideration given to establishing a low-noise environment. Five noise measurement points were set around the reference flight path. Noise levels were recorded at these points using noise measurement devices as shown in Fig. 4. The NED coordinate system with a landing point as the origin was defined. Figure 5 shows a map of the area around the reference path and measurement points. Table 1 indicates the coordinates of the noise measurement points. The initial condition of the flight at $t=0$, which coincides with the moment when the landing approach begins, is given from the real flight situation. The final condition at $t=t_{\mathrm{f}}$, which is also an optimized parameter, is defined in Table 2. The landing approach flights are completed at the final condition. If the helicopter continued to fly at the final flight-path angle from the final position, it would reach the landing point. Thus, $h_{\mathrm{f}}[\mathrm{ft}]$ is calculated from $x\left(t_{\mathrm{f}}\right)[\mathrm{ft}], y\left(t_{\mathrm{f}}\right)[\mathrm{ft}], U\left(t_{\mathrm{f}}\right)[\mathrm{ft} / \mathrm{min}], W\left(t_{\mathrm{f}}\right)[\mathrm{ft} / \mathrm{min}]$ according to the following equation:

$$
h_{\mathrm{f}}=-\sqrt{x\left(t_{\mathrm{f}}\right)^{2}+y\left(t_{\mathrm{f}}\right)^{2}} \frac{W\left(t_{\mathrm{f}}\right)}{U\left(t_{\mathrm{f}}\right)}
$$

The problem of this study was to find a flight trajectory from the initial condition to the final condition which minimizes the noise levels measured at the five measurement points.

\subsection{Performance index}

An energy-based noise estimation index that is widely used for aircraft noise estimation was computed ${ }^{(11)}$. This index is computed by time-integrating the energy of the noise level at each instant. The problem is that there were multiple measurement points in this study. First, the noise level $L_{i}(t)$ of the measurement point $i \quad(i=1,2,3,4,5)$ at distance $r_{i}(t)$ 
from the helicopter at time $t$ was computed using Eqs. (16) and (19).

$$
\begin{aligned}
& L_{i}(t)=L_{\mathrm{ref}}\left(\gamma_{2}(t), \Phi(t)\right)-\operatorname{att}\left(r_{i}(t)\right)[\mathrm{dB}] \\
& \gamma_{2}(t)=\gamma+\frac{180}{\pi} \cdot \frac{\dot{U}(t)}{g} \quad[\mathrm{deg}] .
\end{aligned}
$$

Let $L_{m}(t)$ be the average of the energies of these noise levels. The energy-based noise estimation $E_{A}$ can be computed as follows.

$$
\begin{aligned}
& L_{m}(t)=10 \log \left[\frac{1}{5} \sum_{i=1}^{5} 10^{\frac{L_{i}(t)}{10}}\right] \quad[\mathrm{dB}] \\
& E_{A}=\int_{0}^{t_{\mathrm{f}}} 10^{\frac{L_{m}(t)}{10}} \mathrm{~d} t
\end{aligned}
$$

$E_{A}$ is also expressed as follows:

$$
\begin{aligned}
& E_{A}=\frac{1}{5} \sum_{i=1}^{5} E_{A i}, \\
& E_{A i}=\int_{0}^{t_{f}} 10^{\frac{L_{i}(t)}{10}} \mathrm{~d} t .
\end{aligned}
$$

That is to say, in this paper, the energy-based noise estimation $E_{A}$ was determined by averaging the energies of the noise at the measurement points. The goal of the optimization is to minimize $E_{A}$. Note that $10^{L_{m}(t) / 10}$ and $10^{L_{i}(t) / 10}$ are dimensionless quantities and $E_{A}$ includes a unit of time.

However, if we were to minimize only $E_{A}$ as a performance index, the optimal thrust $T$, which is a control value, would chatter between the upper bound and lower bound, because the performance index does not include the control variables implicitly, and the equations of motion, Eqs. (1)-(6), are linear forms with respect to the thrust $T^{(12)}$. To prevent this chattering, the square integration of the variation of the control values from $T / m g=1$ and $\Theta=\Phi=0$ at a steady level flight was also minimized:

$$
S_{u}=\int_{0}^{t_{\mathrm{f}}}\left\{\left(\frac{T}{m g}-1\right)^{2}+\Theta^{2}+\Phi^{2}\right\} \mathrm{d} t .
$$

Thus, the performance index minimized in this study is defined as

$$
J=E_{A}+w S_{u} .
$$

Here, the units of pitch angle $\Theta$ and roll angle $\Phi$ are radians, and $S_{u}$ has the same unit as $E_{A}$. The weight factor $w$ was a nearly-minimum value within the range where the undesired oscillation of the thrust disappeared.

\section{Real-Time Trajectory Optimization Method}

\subsection{Fundamental concepts}

As a precondition for real-time flight trajectory optimization, the overall trajectory from the present condition to the final condition should always be obtained in order that the pilot, manually controlling, can check the trajectory with the instrument display. The optimization method uses the BDH (Block-Diagonal Hessian) method ${ }^{(13)}$, which can incorporate all kinds of constraint conditions and demonstrates excellent convergence. This method discretizes all of the state variables, control variables, motion equations, and constraint condition equations, which are functions of time, and transforms the optimal control problem, which is a dynamic optimization problem, into a nonlinear programming problem 
that is a static optimization problem. The optimal solution to the converted nonlinear programming problem is computed using a dedicated sparse sequential quadratic programming (SQP) method. A feature of the $\mathrm{BDH}$ method is to discretize the motion equations with the trapezoidal rule. The discretization makes the Hessian in the SQP method a sparse block-diagonal matrix and allows efficient computation. However, though the BDH method can obtain the optimal solution at high speed, it is impossible to compute a highly accurate optimal flight trajectory in real time in response to changes in the flight state. This is because an accurate solution requires a large number of nodal points to discretize the variables and functions. Thus, we added the following two schemes to the BDH method. The fundamental algorithm is the same as that in Ref. 14, 15.

First, the optimization does not strictly follow the transitions of the flight state, but rather a position slightly ahead of the current flight state is set as the initial condition. At some point, the helicopter flies along a tentative optimal trajectory from the existing flight condition to the final condition. While the helicopter is tracing the trajectory, the forthcoming condition is set as the initial condition and the optimal trajectory is recomputed under the latest flight environment and parameters. The recomputation is completed by the time the helicopter reaches the initial condition. The trajectory optimization is iterated in this way. The distance between the actual flight state and the selected initial condition depends on the optimization speed. This method, which can produce a trajectory to the final condition, satisfies the precondition.

Second, if the overall optimal trajectory is computed according to the first scheme, the optimization requires a lot of computation time when the helicopter flies far away from the final condition. This is because a long flight time requires many nodal points and many optimization variables. We therefore allocate the nodal points with some ingenuity to reduce the computation time without losing accuracy. The number of nodal points is held constant regardless of the distance between the helicopter and the final position, and the trajectory is divided into two intervals. Many nodal points are collocated to the interval nearest the present position of the helicopter, while fewer nodal points are collocated to the other interval away from the helicopter. The closer the flight trajectory, the more precise the trajectory obtained. The interval is called a stage in the algorithm.

\subsection{Algorithm}

The real-time optimization algorithm based on the above schemes is presented below. Refer to Fig. 6.

Step 1) First, Stage 0 is defined. The initial state is the helicopter condition at the beginning of the optimal flight. The time length of Stage 0 is $T_{\text {update }}$ seconds. In Stage 0 ,

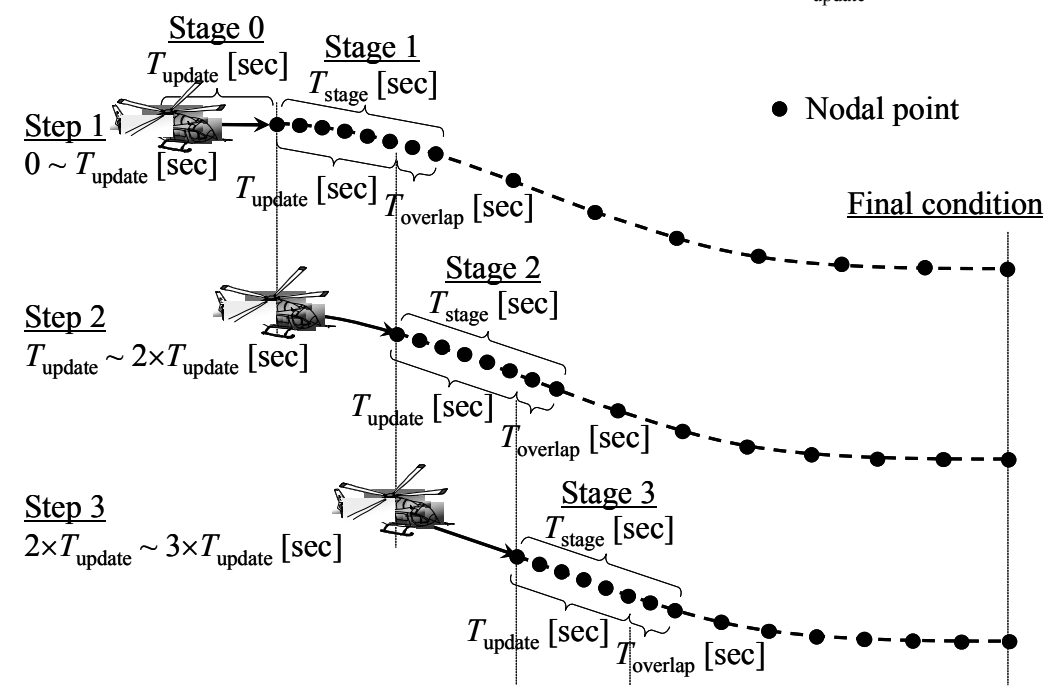

Fig. 6 A reference path and five measurement points. 


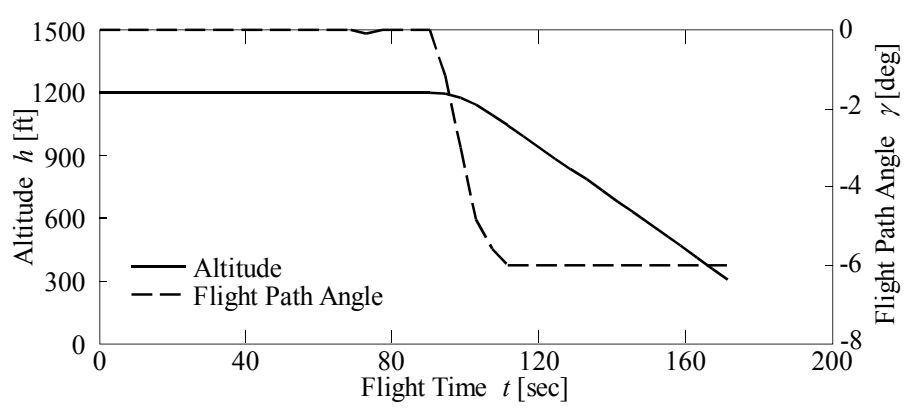

Fig. 7 Initial flight trajectory.

the helicopter flies at a constant altitude and velocity. While the helicopter flies in Stage 0, the flight trajectory from the terminal condition of Stage 0 to the final condition of the overall flight trajectory is optimized. Here, the $T_{\text {stage }}$-second interval from the terminal state of Stage 0 is defined as Stage 1. The number of nodal points discretized in Stage 1 is $N_{1}$, and the number of nodal points discretized between the terminal state of Stage 1 and the final condition of the overall flight trajectory is $N_{2}$. That is to say, the overall flight trajectory is discretized by $N_{1}+N_{2}$ nodal points in total. $N_{1}$ and $N_{2}$ are independent of the distance between the helicopter and the final position. This means that, if the helicopter is far from the final position, the $N_{1}$ nodal points of the first half of the overall flight trajectory are collocated densely and the $N_{2}$ nodal points of the last half are collocated sparsely.

The optimal trajectory obtained in the BDH method depends on an initial guess as to the solution. The reference path shown in Fig. 5 is the initial guess. Figure 7 indicates the altitude and flight path angle of a reference path. The airspeed keeps constant at $70 \mathrm{kt}$ up to the final condition. The helicopter flies at constant altitude and velocity and reaches the final position in a descent flight at a flight path angle of $-6 \mathrm{deg}$. This is considered a normal approach. Since the generated optimal flight trajectory is indicated to the pilots with the display instruments, the optimization is stopped either when the solution converges near the optimal solution or the calculation time reaches $T_{\text {update }}$ seconds.

Step 2) Entering Stage 1, the helicopter starts to fly along the flight trajectory obtained in Step 1 by the pilot control. While the helicopter flies within the first $T_{\text {update }}$ seconds in Stage 1, the flight trajectory from Stage 2 to the final condition is optimized. Stage 2 is defined as a $T_{\text {stage }}$-second interval, of which the initial state is the state at $T_{\text {update }}$ seconds after the beginning of Stage 1 obtained in Step 1. Here, Stage 2 overlaps the last $T_{\text {overlap }}$-second interval of Stage 1. The overlapping connects the trajectories smoothly. In Stage 2, the number of nodal points near the present position is $N_{1}$, and the number of nodal points between the terminal state of Stage 2 and the final condition is $N_{2}$, the same as in Step 1. That means the flight trajectory roughly optimized in the previous step is reoptimized with higher accuracy. It should be noted that the optimal trajectory optimized in Step 1 can be utilized as the initial guess in this step. Since the optimal flight trajectory has already been obtained roughly in Step 1, the optimization converges in a comparatively short time. Before the $T_{\text {update }}$-second flight in Stage 2, the optimization is stopped and the flight trajectory displayed on the instrument is updated.

Step 3) In a similar way as described in Step 2, while the helicopter flies in the first $T_{\text {update }}$ seconds of Stage 2 along the flight trajectory generated in Step 2, the flight trajectory from Stage 3 to the final condition is optimized.

Step 4) The above-mentioned step is repeated until the helicopter gets close to the final condition. If the remaining flight time to the final condition is within $T_{\text {final }}$ seconds, the overall flight trajectory is discretized with $N_{1}+N_{2}$ nodal points and optimized at once.

In the optimization, we should consider wind effects; otherwise, it is very difficult for pilots to follow the optimized flight trajectory. MuPAL- $\varepsilon$ used in this study can measure three-axis airspeed with air data sensors and ground speed with a high-precision GPS/INS. 
Thus, the wind velocity is estimated from the difference between the ground speed and airspeed. The average values of the wind velocity components are calculated in one step and substituted for Eqs. (1) and (2) in the motion equations in the next step. By repeating this process, the averaged wind effect is incorporated with the $T_{\text {update }}$-second delay. This paper does not consider the wind effect on the noise level.

\section{Numerical Simulations}

\subsection{Computation condition}

We conducted numerical simulations to confirm the effectiveness of the real-time flight trajectory optimization. The real-time optimization was compared with an offline optimization. The offline optimization optimized the entire flight trajectory over a long time. In the real-time optimization, we used a personal computer with an Intel Core 2 Duo T7200 (2GHz) CPU. The optimization parameters, which are determined from the computer performance, are summarized in Table 3. The initial condition was defined in Table 4. We assume that there was no wind; i.e., $u_{g}=0$.

\subsection{Comparison with offline optimization}

Figure 8 shows the optimal trajectories. The noise source model used in this study reached its maximum value when the flight path angle was around -5 deg as shown in Fig. 2. Optimal solutions therefore attempted to avoid this flight path angle. In addition, since the performance index includes the time-integral of the noise, the flight time is likely to be shortened. The helicopter starts to descend at the maximum descent rate ( $800 \mathrm{fpm})$ after a level flight in about 80 seconds. When the level changes to the descent, the flight path angle passes through the vicinity of -5 deg as quickly as possible in order to avoid loud noise. If the descent rate was $800 \mathrm{fpm}$ and the horizontal velocity was $100 \mathrm{kt}$, which was the initial velocity, the flight path angle would be $-4.5 \mathrm{deg}$, which would cause a loud noise. Thus, the helicopter decreases the horizontal velocity to the minimum velocity of $50 \mathrm{kt}$. This allows for a steeper descent at a flight path angle of less than $-9.0 \mathrm{deg}$. In the offline optimization, since a shorter flight time decreases the performance index value, the helicopter flies at an initial velocity of $100 \mathrm{kt}$ for a while after the flight begins, and, when starting the descent, decreases its velocity at the maximum deceleration of Eqs.(12) and (13). In the latter half of the flight, the helicopter keeps at the maximum sink rate to avoid producing loud noise at low altitudes. The flight changes from the level to the descent when the descending flight leads exactly to the final point. On the other hand, in terms of lateral movement, the helicopter has to turn frequently in order to avoid the noise measurement points on the ground. Accordingly, the bank angle changes significantly.

There is a noticeable difference between the offline optimization and real-time optimization in the velocity profile. In the real-time optimization, the helicopter decelerates after starting the flight. This deceleration is contrary to the logic of the offline optimization. This discrepancy is caused by the difference in the number of nodal points. Since the number of nodal points in the real-time optimization is smaller, the real-time optimization has a computation error.

Table 3 Parameters of real-time optimization

\begin{tabular}{|c|c|}
\hline Parameter & Value \\
\hline$N_{1}$ & 10 \\
\hline$N_{2}$ & 10 \\
\hline$T_{\text {stage }}$ & $40 \mathrm{~s}$ \\
\hline$T_{\text {update }}$ & $30 \mathrm{~s}$ \\
\hline$T_{\text {overlap }}$ & $10 \mathrm{~s}$ \\
\hline$T_{\text {final }}$ & $90 \mathrm{~s}$ \\
\hline
\end{tabular}

Table 4 Initial condition

\begin{tabular}{|c|c|}
\hline Variable & Initial condition \\
\hline$x, \mathrm{n} \mathrm{mile}$ & -2.70 \\
\hline$y, \mathrm{n} \mathrm{mile}$ & -2.37 \\
\hline$h, \mathrm{ft}$ & 1200 \\
\hline$U, \mathrm{kt}$ & 100 \\
\hline$W, \mathrm{ft} / \mathrm{min}$ & 0 \\
\hline$\Psi, \mathrm{deg}$ & free \\
\hline
\end{tabular}




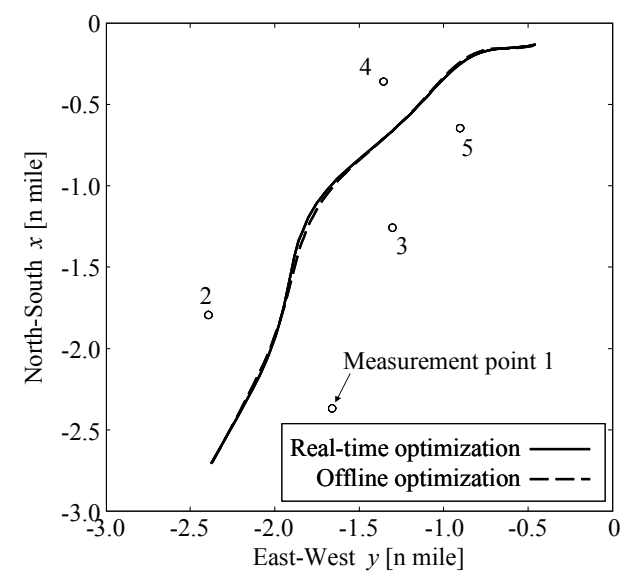

a) Flight trajectories on the horizontal plane.

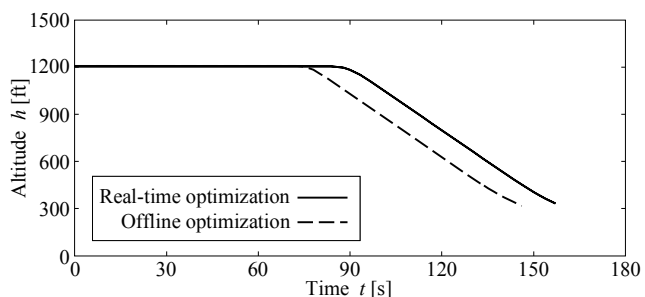

b) Altitude histories.

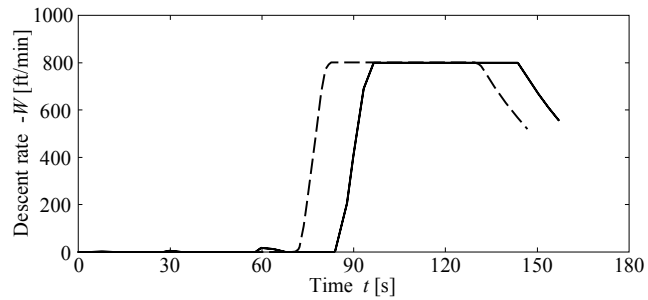

d) Descent rate histories.

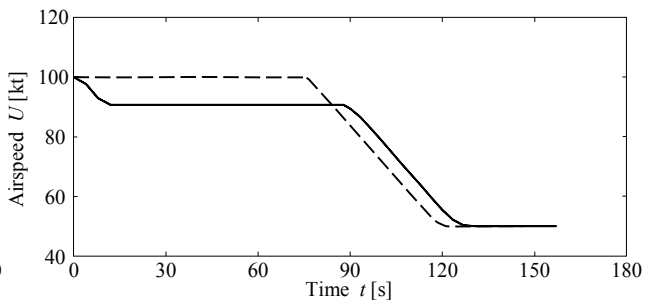

c) Horizontal airspeed histories.

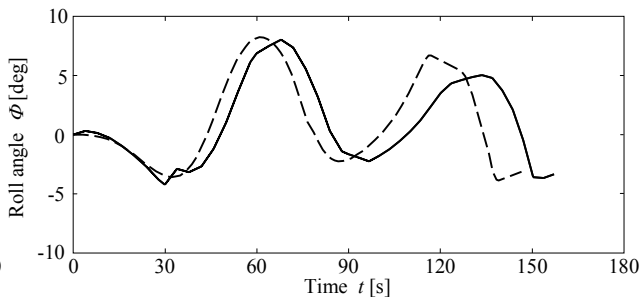

e) Roll angle histories.

Fig. 8 Flight trajectories of real-time and offline optimization.

Table 5 Noise levels of the optimal flights

\begin{tabular}{|c|c|c|c|}
\hline Trajectory case & $\begin{array}{c}\text { Flight time } \\
t_{\mathrm{f}}, \mathrm{s}\end{array}$ & $\begin{array}{c}\text { Energy-based noise } \\
\text { estimation } E_{A}\end{array}$ & $\begin{array}{c}\text { Temporal-mean noise } \\
\text { level } L_{A}, \mathrm{~dB}\end{array}$ \\
\hline Real-time optimization & 157.2 & $5.01 \times 10^{7}$ & 55.0 \\
\hline Offline optimization & 147.4 & $4.90 \times 10^{7}$ & 55.2 \\
\hline
\end{tabular}

Table 5 shows the flight time $t_{\mathrm{f}}$, the energy-based noise estimation $E_{A}$, and the temporal-mean noise level $L_{A}$ of both optimizations. This temporal-mean noise level is a quantity expressing the time-average of the energy-based noise estimation $E_{A}$. That is to say, it is calculated as:

$$
L_{A}=10 \log \left(\frac{E_{A}}{t_{\mathrm{f}}}\right)=10 \log \left(\frac{1}{t_{\mathrm{f}}} \int_{0}^{t_{\mathrm{f}}} 10^{\frac{L_{m}(t)}{10}} \mathrm{~d} t\right) \quad[\mathrm{dB}] .
$$

The energy-based noise estimation is larger in the real-time optimization than in the offline optimization, but the difference is not particularly large. On the contrary, the temporal-mean noise level of the real-time optimization is slightly smaller than that of the offline optimization due to the flight times.

\subsection{Noise reduction effect}

This subsection proves the effectiveness of noise abatement by the real-time optimal flight in the numerical simulations. The noise of the real-time optimal flight is compared with those of the following two conventional landing approaches. The one is a 6-degree 
Table 6 Noise levels of conventional flights

\begin{tabular}{|c|c|c|c|}
\hline Trajectory case & Flight time $t_{\mathrm{f}}, \mathrm{s}$ & $\begin{array}{c}\text { Energy-based noise } \\
\text { estimation } E_{A}\end{array}$ & $\begin{array}{c}\text { Temporal-mean noise } \\
\text { level } L_{A}, \mathrm{~dB}\end{array}$ \\
\hline 6 degree descent & 171.5 & $8.71 \times 10^{7}$ & 57.1 \\
\hline 3 degree descent & 171.6 & $1.07 \times 10^{8}$ & 58.0 \\
\hline
\end{tabular}

descent approach trajectory, which is the same as the reference trajectory shown in Figs. 5 and 7, and the other is a 3-degree descent approach trajectory where the final flight-path angle of the former 6-degree descent approach is changed to -3 degrees. The 3-degree descent approach simulates an ILS (Instrument Landing System) approach used for fixed-wing aircraft. The ILS approach is proposed recently for helicopter operations in bad atmospheric conditions such as rain and fog.

The maximum noise levels of the real-time optimal flight at the measurement points are smaller than those of the conventional flights because the moderate flight path angles in the conventional approaches cause strong BVI noise. In particular, prominent differences of the noise levels appear at measurement points 3, 4 and 5. Table 6 shows the flight time $t_{\mathrm{f}}$, the energy-based noise estimation $E_{A}$, and the temporal-mean noise level $L_{A}$ of the conventional flights. From the comparison of Tables 5 and 6 , the flight time and the temporal-mean noise levels of the optimal flights are both less than those of the conventional approach flights.

\section{Flight Experiments}

\subsection{System configurations}

In order to confirm the effectiveness of the proposed optimization techniques, we conducted real flight experiments. As stated previously, the flight experiments were performed at Taiki Multi-Purpose Aeronautical Park using the JAXA-owned MuPAL- $\varepsilon$. The pilot controlled all flights manually. In order to track the indicated trajectories exactly, the flight profile was shown to the pilot using an experimental display on an instrument panel, which is called "Tunnel-in-the-Sky" (16). This display indicated speed commands as well as the flight trajectory. The optimal flights obtained in this paper differ from the conventional approaches, but, in the flight simulation experiment before the real flight, the pilot commented that it was not difficult to track the optimal trajectory with this display. In the flight experiments, a landing approach that the pilot judged to be normal flight was also conducted in order to compare it with the optimal flights.

In the vicinity of an airport, the acceptable noise level usually depends on the distance from the runway. The noise criterion level at noise measurement point 5 , the measurement point closest to the runway, was awarded an extra allowance of $5 \mathrm{~dB}$ in this study. More specifically, Eq. (23) was rewritten as

$$
\begin{aligned}
L_{m}(t) & =10 \log \left[\frac{1}{5} \sum_{i=1}^{5} 10^{\frac{L_{i}(t)-L_{\text {allow }}}{10}}\right] \quad[\mathrm{dB}], \\
L_{\text {allow } i} & =\left\{\begin{array}{ll}
0 & (i=1, \ldots, 4) \\
5 & (i=5)
\end{array}[\mathrm{dB}] .\right.
\end{aligned}
$$

The parameters of the real-time optimization have the same values as in Table 3. 


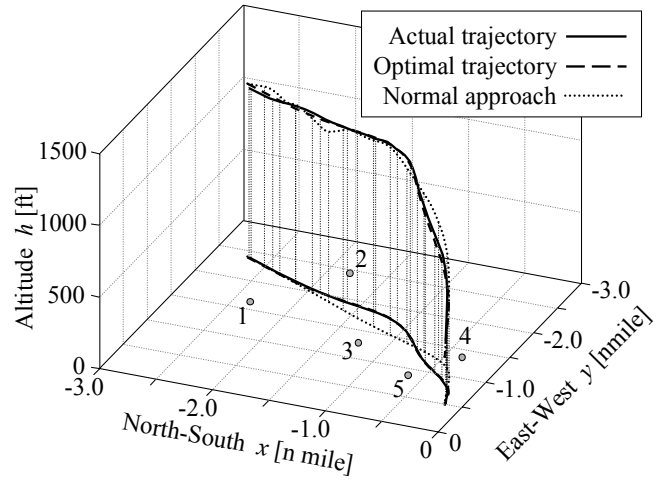

a) Flight trajectories.

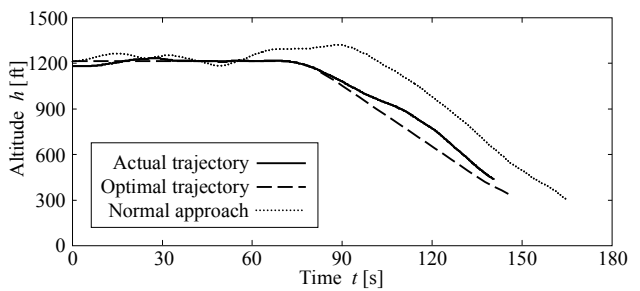

b) Altitude histories.

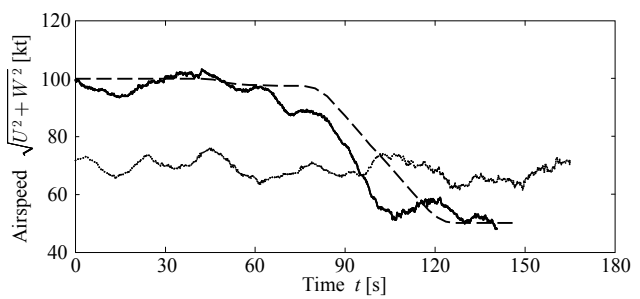

c) Horizontal airspeed histories.

Fig. 9 Flight trajectories.

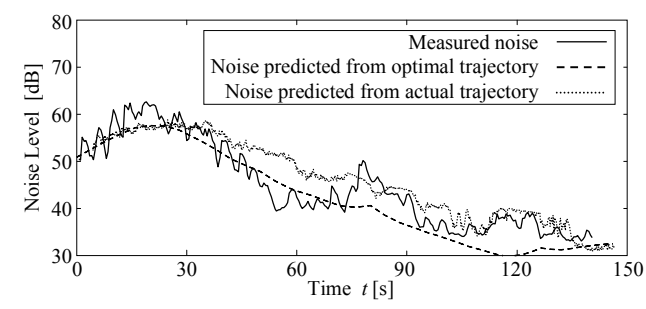

a) Measurement point 1

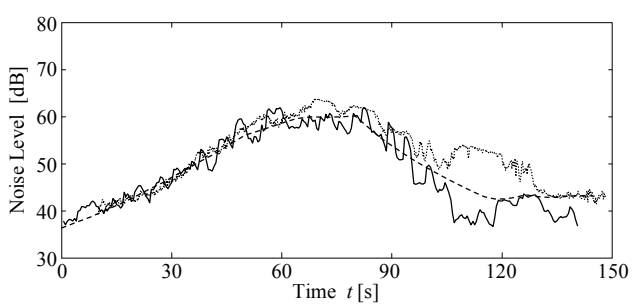

c) Measurement point 3

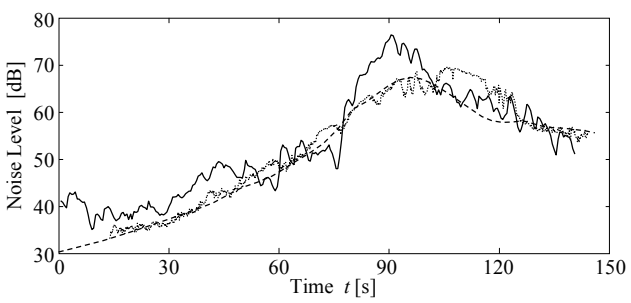

e) Measurement point 5

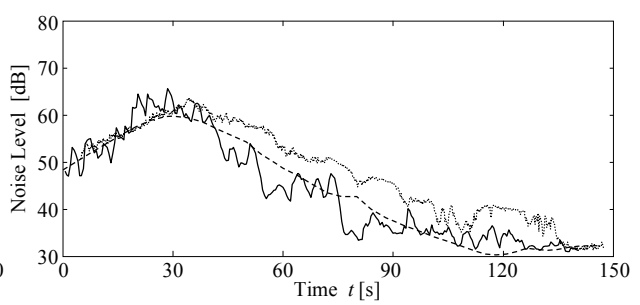

b) Measurement point 2

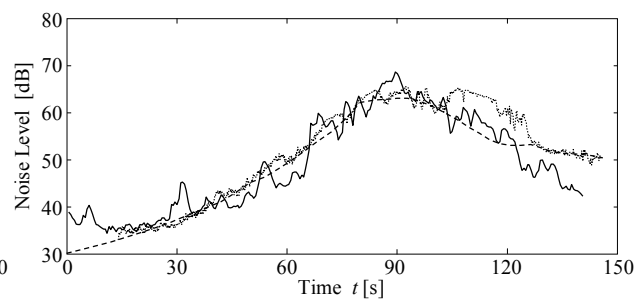

d) Measurement point 4

Fig. 10 Noise levels in the optimal flight.

\subsection{Flight experiments results}

The flight data obtained using MuPAL- $\varepsilon$ 's measurement systems are shown in Fig. 9. This is an example of some experiments. The initial condition in which the helicopter started to fly was almost the same as the numerical simulation of the previous section. The proposed real-time trajectory optimization algorithm worked well even in a real flight environment. The comparison of the optimal trajectory indicated to the pilot and the real flight trajectory suggests that the airspeed and flight path angle had vibrations. There was a tracking error in the descent flight. However, the constraint conditions of the computational optimization in Eqs. (7)-(13) were defined appropriately. In the future, it will probably be necessary to determine an optimal solution (a robust optimal solution) that suppresses the additional noise due to tracking errors. According to the pilot, it was difficult to match the 


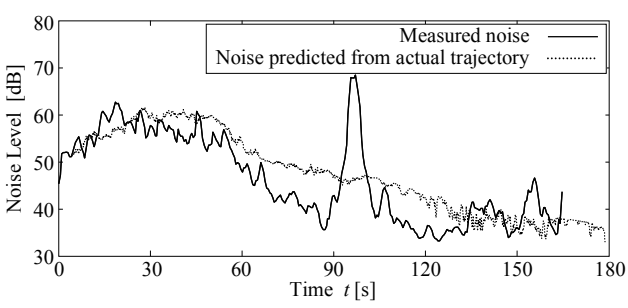

a) Measurement point 1

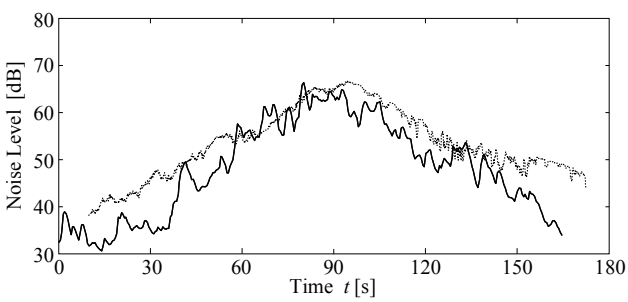

c) Measurement point 3

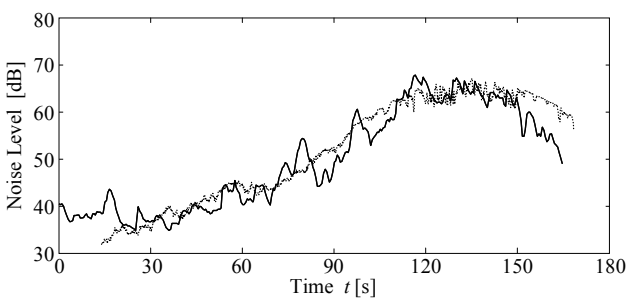

e) Measurement point 5

Fig. 11 Noise levels in the normal approach.

Table 7 Flight experiment results

\begin{tabular}{|c|l|c|c|}
\hline Trajectory case & \multicolumn{1}{|c|}{ Noise type } & $\begin{array}{c}\text { Flight time } \\
t_{\mathrm{f}}, \mathrm{s}\end{array}$ & $\begin{array}{c}\text { Temporal-mean noise } \\
\text { level } L_{A}, \mathrm{~dB}\end{array}$ \\
\hline \multirow{2}{*}{ Optimal flight } & Measurement & 146.1 & 54.0 \\
\cline { 2 - 4 } & $\begin{array}{l}\text { Prediction from } \\
\text { optimal trajectory }\end{array}$ & 140.8 & 56.3 \\
\cline { 2 - 4 } & $\begin{array}{l}\text { Prediction from } \\
\text { actual trajectory }\end{array}$ & 140.8 & 56.6 \\
\hline Normal approach & Measurement & 165.0 & 57.3 \\
\cline { 2 - 4 } & $\begin{array}{l}\text { Prediction from } \\
\text { actual trajectory }\end{array}$ & 165.0 & 56.6 \\
\hline
\end{tabular}

airspeed and climb rate perfectly because he was confused regarding the timing of their changes, but it was not difficult to make the vehicle position track the optimal solution. In the normal approach, the approaching speed became smaller and the helicopter turned and descended near the final position. In general, the pilot did not change the vehicle's attitude more than necessary so as to pay attention to the surroundings.

Figures 10 and 11 show the noise levels actually recorded at the measurement points. The difference in the noise levels of the optimal flight and the normal approach was small. The figures include the noise levels predicted from the actual flight data, the noise source model and the attenuation model. The figures also show the noise level predicted in the case where the helicopter flies exactly along the optimal trajectory. A comparison between the measured noise levels and the noise levels predicted from the actual trajectory reveals the precision of the noise source and attenuation models. The noise levels could be predicted within about $10 \mathrm{~dB}$ error. Figure 10 indicates that the noise levels actually measured and predicted from the optimal trajectory had a significant difference in peak value at the noise measurement point 5. The possible causes of this discrepancy are tracking errors, the 
directionality of helicopter noise, and the effect of wind on noise propagation. In addition, spike-like noises appear, especially in Fig. 11 a). They may emanate from cars passing near the measurement points. The predicted levels tended to correspond with the measured levels except for the spike-like noises. Table 7 summarizes the results of the flight experiments and the optimal solutions. The temporal-mean noise level of the optimal flight was $3.3 \mathrm{~dB}$ smaller than that of the normal landing approach. However, the difference in the temporal-mean noise levels predicted from the actual flight and those really measured indicated that the noise model has error of 0.7-2.6 dB. Therefore, the results do not necessarily show a clear effect of noise reduction.

\section{Conclusions}

This research generated flight profiles for helicopter landing approaches that reduce the ground noise using computational optimization techniques. The optimal control problem was first defined according to the flight test environment under relatively simple helicopter dynamics and noise models. Then, a new optimization algorithm was proposed in order to conduct real-time flight trajectory optimization that can deal with changes in the real environment. The obtained optimal trajectories are characterized by a short flight time and a steep descent angle, which avoids strong noise. The optimal trajectories also attempted to avoid the measurement points, at which the noise levels should be minimized. In the numerical simulations, the trajectory optimized in real time differed slightly from that of the offline optimization, but the estimated noise levels were almost equal. Furthermore, flight experiments were conducted. The real-time trajectory optimization worked well even in a real flight environment. The results show differences between the predicted noise levels and the actually measured noise levels because of background noise and tracking errors in the real environment. However, the tendency of the actually measured noise levels agreed with that of the predicted noise levels, and the noise of the optimal flights was slightly lower than that of the normal landing approach.

The real-time optimized flight resulted in only a small benefit of noise abatement. The authors will therefore update the noise source model to incorporate directionality, and the noise attenuation model to include external disturbances. The performance index should be changed into an index that reflects the unpleasantness of the noise more appropriately. Moreover, we plan to improve the optimization algorithm, and increase the number of noise measurement points to develop more precise and practical low-noise approaches considering actual land use.

\section{Acknowledgments}

This research was carried out as a collaboration between the University of Tokyo and JAXA.

\section{References}

(1) Funabiki, K., Muraoka, K., Iijima, T., and Shiomi K., NOCTARN: Trajectory Based CNS/ATM Concept for Small Aircraft, 21st Digital Avionics Systems Conference, (2002), pp. 485-492.

(2) Lucas, M. J., and Marcolini, M. A., "Rotorcraft Noise Model," American Helicopter Society Technical Specialists' Meeting for Rotorcraft Acoustic and Aerodynamics, Williamsburg, VA, 1997.

(3) Gopalan G., Sim B. W., and Schmitz F. H., "Flight Path Management and Control Methodology to Reduce Helicopter Blade-Vortex Interaction (BVI) Noise," American 
Helicopter Society Vertical Lift Aircraft Design Conference, San Francisco, California, 2000.

(4) Min, Xue, and Ella, M. A., "Noise-Minimum Runway-Independent Aircraft Approach Design for Baltimore-Washington International Airport," Journal of Aircraft, Vol. 43, No. 1, 2006, pp. 39-51.

(5) Ishii, H., Okuno, Y., and Funabiki, K., "Flight Experiments for Aircraft Noise Measurement using “Tunnel-in-the-Sky” Display,” AIAA Paper 2002-4880, 2002.

(6) Ishii, H., Gomi, H., and Okuno, Y., "Helicopter Noise Tests for BVI Noise Measurement Using an Onboard External Microphones,” AIAA Paper 2005-6119, 2005.

(7) Okuno, Y., and Matayoshi, N., "Development of a New Research Helicopter MuPAL-E," 57th American Helicopter Society Annual Forum, Washington DC, 2001.

(8) Tsuchiya, T., Ishii, H., Uchida, J., Gomi, H., Matayoshi, N., and Okuno, Y., "Optimal Flight for Ground Noise Reduction in Helicopter's Landing Approach: Optimal Altitude and Velocity Control," Transactions of the Japan Society for Aeronautical and Space Sciences, Vol. 50, No. 169, 2007, pp. 209-217.

(9) Tsuchiya, T., Ishii, H., Uchida, J., Ikaida, H., Gomi, H., Matayoshi. N., and Okuno, Y., "Flight Trajectory Optimization to Minimize Ground Noise in Helicopter Landing Approach," Journal of Guidance, Control, and Dynamics, Vol. 32, No. 2, 2009, pp. 605-615.

(10) ISO 9613-1, 1st ed., Acoustics - Attenuation of Sound during Propagation Outdoors - Part 1: Calculation of the Absorption of Sound by the Atmosphere, International Organization for Standardization, 1993.

(11) Yoshioka, H., Iwasaki, K., and Yamada, I., "Recent Development on Energy-Based Aircraft Noise Modeling in Japan," 18th International Congress on Acoustics [CD-ROM], 2004.

(12) Bryson, A. E., Jr. and Ho, Y. C. Applied Optimal Control, Hemisphere Publishing Company, NY, 1975.

(13) Yokoyama, N., Suzuki, S., and Tsuchiya, T., "Convergence Acceleration of Direct Trajectory Optimization Using Novel Hessian Calculation Methods," Journal of Optimization Theory and Applications, Vol. 136, No. 3, 2008, pp. 297-319.

(14) Tsuchiya, T., Miwa, M., Suzuki, S., Masui, K., and Tomita, H., "Real-Time Flight Trajectory Optimization and Its Verification in Flight," Journal of Aircraft, Vol. 46, No. 4, 2009, pp. 1468-1471.

(15) Miwa, M., Tsuchiya, T., Yonezawa, S., Yokoyama, N., and Suzuki, S., "Real-Time Flight Trajectory Generation Applicable to Emergency Landing Approach," Transactions of the Japan Society for Aeronautical and Space Sciences, Vol. 52, No. 175, 2009, pp. 21-28.

(16) Funabiki, K., "Design of Tunnel-in-the-Sky Display and Curved Trajectory," 24th International Congress of the Aeronautical Science [CD-ROM], ICAS 2004-8.4.4, 2004. 